\title{
Postoperative acute heart failure in an 18-year-old McCune-Albright syndrome patient without preoperative cardiac morphology abnormality - a case report and literature review
}

\author{
Rongjie $\mathrm{Wu}^{1}$ and Guangtao $\mathrm{Fu}^{1}$ \\ ${ }^{1}$ Guangdong Provincial People’s Hospital
}

June 1, 2021

\begin{abstract}
An 18-year-old female patient diagnosed with McCune-Albright syndrome (MAS) developed postoperative acute heart failure with negative findings during preoperative cardiac screening and restricted fluid infusion. Latent cardiovascular involvement of MAS could be a reasonable explanation for the present case.

Postoperative acute heart failure in an 18-year-old McCune-Albright syndrome patient without preoperative cardiac morphology abnormality - a case report and literature review

Rongjie $\mathrm{Wu}^{1,2}$, Guangtao $\mathrm{Fu}^{1}{ }^{1}$ *

1 Department of Orthopedics, Guangdong Provincial People's Hospital, Guangdong Academy of Medical Sciences, Guangzhou, Guangdong Province, P.R.China

2 Shantou University Medical College, Shantou, China.

* indicated corresponding author.
\end{abstract}

\section{Corresponding author}

1\# Guangtao Fu

E-mail: fuguangtao@gdph.org.cn

Tel: $+86-15112160683$

Address: 106, Zhongshan Road, Yuexiu District, Guangzhou, Guangdong Province, P.R.China.

\begin{abstract}
An 18-year-old female patient diagnosed with McCune-Albright syndrome (MAS) developed postoperative acute heart failure with negative findings during preoperative cardiac screening and restricted fluid infusion. Latent cardiovascular involvement of MAS could be a reasonable explanation for the present case.
\end{abstract}

\section{INTROUCTION}

McCune-Albright syndrome (MAS) is a rare genetic disease characterized by polyostotic fibrous dysplasia, skin hyperpigmentation, and endocrine dysfunction, with the prevalence ranging between 1/100 000 and $1 / 1000000^{1,2}$. Due to the severe deformity of lower limbs caused by fibrous dysplasia in the proximal femur or tibia, substantial numbers of patients diagnosed with MAS are admitted to orthopedics clinics for surgical interventions ${ }^{3}$. Although the skeletal and endocrine abnormalities of MAS are well recognized by 
most clinicians, rare but fatal cardiovascular involvement is seldom reported. It is also reported that cardiac abnormality such as myocyte hypertrophy, visible microscopically in a macroscopically normal heart, strongly suggests that Gs mutation may be present in the heart with inconspicuous macroscopic findings ${ }^{4}$. Thus, the cardiovascular involvement of MAS patients is prone to be overlooked in clinical practice and related to potential risk of medical disputes. To the best of our knowledge, cardiovascular involvement of MAS patients who underwent orthopedic surgery has not yet been presented. Here, we report the case of a young woman with MAS who suffered from acute heart failure after proximal femur osteotomy for lower limb deformity correction, although she had no positive cardiovascular abnormality or history of cardiovascular disease.

\section{CASE PRESENTATION}

\subsection{History of present illness}

An 18-year-old woman who suffered from left lower limb deformity and thigh pain for $>10$ years was admitted to our center. Physical examination revealed lower limb discrepancy $(-2.5 \mathrm{~cm}$ in left leg), limping gait, left groin area tenderness, and restricted range of motion in the left hip. Neither café-au-lait spots nor sexual precocity was found. X-ray and computed tomography (CT) imaging showed severe coxa varus in the left hip (femoral neck-shaft angle $=60^{\circ}$ ), varus alignment of the femur, and shepherd cane deformity (Figure 1A). Single-photon emission computed tomography (SPECT) demonstrated multiple osteolytic lesions located in craniofacial bone, left femur and left tibia (Figure 1B). Preoperative electrocardiography and chest radiography showed no positive findings. Polyostotic fibrous dysplasia was considered as the primary diagnosis. For lower limb deformity correction, proximal femur osteotomy and internal fixation of the left femur were performed after detailed preoperative planning (Figure 2A). Total operation time was 117 min and intraoperative blood loss was $800 \mathrm{~mL}$. Postoperative radiography showed significant improvement of coxa varus and correction of the shepherd's crook deformity (femoral neck-shaft angle $=104^{\circ}$ ) (Figure $2 \mathrm{~B}$ ). Total fluid replacement volume was $1850 \mathrm{ml}$ in the first $24 \mathrm{~h}$ after surgery with restricted fluid infusion rate (40-60 drops / min). Genetic testing of the pathological bone tissue from the proximal left femur revealed mutation of c.601C $>\mathrm{T}$ (Figure 3). The patient was finally diagnosed with MAS according to previous studies $^{5,6}$. However, on the morning of postoperative day 2 , the patient suddenly complained of chest pain, palpitation, and dyspnea without any known cause.

\subsection{History of past illness}

There was no previous medical history.

\subsection{Personal and family history}

No abnormalities.

\subsection{Physical examination}

The patient presented dyspnea without chest pain and fever. Moist rales were heard in both lung fields. High concentration oxygen therapy $(4 \mathrm{~L} / \mathrm{min})$ by mask did not effectively relieve the above symptoms, with the lowest oxygen saturation being $85 \%$.

\subsection{Laboratory examinations}

Blood tests showed significantly increased plasma brain natriuretic peptide (BNP $2600 \mathrm{pg} / \mathrm{mL}$, normal range: $0-125 \mathrm{pg} / \mathrm{mL}$ ), myocardial enzymes (high sensitivity cardiac troponin $\mathrm{T}$ (hs-cTnT) $=27.7 \mathrm{pg} / \mathrm{mL}$, normal range: $0-14 \mathrm{pg} / \mathrm{mL}$ ) and D-dimer $(1670 \mathrm{ng} / \mathrm{mL}$, normal range: 0-500 ng/mL). Other routine blood, biochemistry, liver function and blood coagulation tests were normal.

\subsection{Imaging examinations}

CT showed bilateral lung exudation and pleural effusion (Figure 4). Spiral CT pulmonary angiography showed no abnormality. Echocardiography showed mild tricuspid regurgitation with elevated pulmonary artery systolic pressure $(33 \mathrm{mmHg})$. No clinically significant abnormality was found by emergency electrocardiography (ECG). 


\section{FINAL DIAGNOSIS}

Based on the clinical signs and elevated BNP, the patient was diagnosed with acute heart failure clinically ${ }^{7}$, while pulmonary embolism was excluded based on the results of spiral CT pulmonary angiography.

\section{TREATMENT}

The patient was transferred from the ward to the intensive care unit and noninvasive positive-pressure ventilation, diuresis, and anti-infective therapy were administered immediately.

\section{OUTCOME AND FOLLOW-UP}

On the morning of postoperative day 3 , all the symptoms were significantly relieved, with the oxygen saturation increasing to $100 \%$. Plasma BNP, hs-cTnT and D-dimer levels also returned to normal. Echocardiography also showed no abnormality at this time.

\section{DISCUSSION}

MAS is a rare noninherited genetic disease characterized by polyostotic fibrous dysplasia, café-au-lait spots, and sexual precocity, which was first described by McCune and Bruch and by Albright ${ }^{8}$. In recent years, endocrine abnormalities including hyperthyroidism, growth hormone excess, and Cushing syndrome, as well as nonendocrine abnormalities such as cardiac and hepatic diseases have also been reported ${ }^{1,9}$. Based on the genetic analysis of MAS patients, the mutation of guanine nucleotide-binding protein $\alpha$-stimulating activity polypeptide (GNAS) is widely accepted as the major pathological mechanism of MAS ${ }^{10}$. The most common mutation site of GNAS is the amino acid Arg 201, which is replaced by Cys or His or Ser or Gly ${ }^{6,10,11}$. It is reported that the mutation of GNAS affects somatic cells but not genital cells, while the number of somatic cells mutated in specific tissues and organs varies ${ }^{11-14}$. Thus, MAS patients could present with variable clinical manifestations, which depend on the location, cell type and numbers of the mutated cells ${ }^{4,9,15}$.

Polyostotic fibrous dysplasia is the main skeletal manifestation of MAS, ranging from $46 \%$ to $98 \%$ according to different reports ${ }^{4}, 16$. The femur and craniofacial region are the most commonly affected. Most of the patients with MAS have at least one fracture, and even multiple fractures, especially between 6 and 12 years old ${ }^{4}$. In addition to bone fractures, fibrous dysplasia in the appendicular skeleton can lead to pain, limping, and deformities of the weight-bearing bone, while fibrous dysplasia in the craniofacial bones can lead to painless lumps or facial asymmetry. Besides, compressive syndrome and scoliosis caused by fibrous dysplasia have also been reported ${ }^{2}$. As the progression of fibrous dysplasia may bring about deformities as well as physical impairment such as limping and the need for a wheelchair, orthopedic surgical intervention is usually required for the recovery of motor functions ${ }^{2,3}$.

Cardiovascular involvement, as a rare event in MAS patients, is one of the fatal conditions that can present as tachycardia, hypertension, cardiac hypertrophy, and even sudden death ${ }^{4,9}$. Only one study has reported heart failure as the manifestation of cardiac involvement ${ }^{17}$. Previously, cardiac abnormalities in patients with MAS were mostly interpreted as a complication of endocrine disturbances ${ }^{9,17}$. Subsequently, hypertrophied myocytes with Gs mutation in the macroscopically normal heart of MAS patients diagnosed with sudden death at autopsy illustrated that the mutation of heart tissue is a more convincing explanation for cardiac manifestations in MAS, rather than the complication of endocrine abnormalities ${ }^{4,9}$. However, the underlying mechanism of cardiovascular involvement in MAS is not entirely clear. It is reported that mutation of GNAS can cause overproduction of cyclic AMP and enhanced phosphorylation of calcium channels, which subsequently increases the $\mathrm{Ca}^{2+}$ permeability of myocardial cells. As a result of intracellular $\mathrm{Ca}^{2+}$ accumulation, cardiac myocytes are continuously overactivated and cause increased heart rate and myocardial contractility, which eventually induce cardiac dysfunction ${ }^{[4,20]}$.

According to the acute heart failure assessment reported by the literature, our patient could be diagnosed with acute heart failure based on elevated NT-proBNP, interstitial edema, dyspnea and absence of fever ${ }^{18}$. For differential diagnosis, pulmonary embolism has been excluded through Spiral CT pulmonary angiography. Normal ECG and no chest pain also do not agree with acute coronary syndrome or arrhythmia due to 
reduced hemoglobin postoperatively. Pulmonary complications were not consistent with extremely high BNP. Considering Swan-Ganz catheter or nuclear imaging is only recommended for non-classical acute heart failure like without BNP elevated or the treatment is not effective ${ }^{19}$, we did not perform them on our patient.

To the best of our knowledge, postoperative acute heart failure in a young patient without a history of cardiac disease and cardiac morphology abnormality has not yet been reported. In consideration of the fact that macroscopic findings of the Gs mutated heart are often inconspicuous in the clinic $^{4}$, as well as the impossibility of acute heart failure for a patient without a history of cardiovascular abnormalities at such a young age, latent cardiovascular involvement of MAS seems to be a reasonable interpretation for the acute heart failure of the present patient.

We proposed two potential mechanisms for acute heart failure in this young patient with limited fluid replacement volume based on the known cardiotoxicity of cAMP and $\mathrm{Ca}^{2+20}$. Firstly, long-term cAMP overload may induce myofilament calcium desensitization of the cardiac myocytes through increased protein kinase A. Therefore, the desensitized cardiac myocytes cannot respond effectively to the increased calcium influx to enhance the cardiac contractibility when the fluid load increases ${ }^{21,22}$. Secondly, long-term $\mathrm{Ca}^{2+}$ overload may have a negative impact on the compliance of the heart due to the hypertrophied myocytes and increased myocardial contractility ${ }^{23}$. However, we admit that further investigation is required to explore further the exact mechanism.

The present report was limited because we could not perform genetic analysis of cardiomyocytes due to regulatory and ethical concerns.

\section{CONCLUSIONS}

Many patients with MAS are admitted to orthopedic departments for surgical intervention. Therefore, it is important for orthopedic surgeons to be aware of polyostotic fibrous dysplasia and other rare but fatal manifestations, such as cardiac involvement. To the best of our knowledge, our report represents the first case of a young woman with MAS and acute heart failure after proximal femur osteotomy, with no cardiovascular abnormality or history of cardiovascular disease. As latent cardiovascular involvement of MAS was considered to be the major cause of this case, we recommend more detailed preoperative cardiovascular screening for MAS patients. We believe that this case report and the literature review will help orthopedic surgeons make a more reasonable prognostic assessment, and subsequently reduce the risk of medical dispute by further understanding this rare but fatal event of MAS.

\section{Declarations}

\section{Ethics approval and consent to participate}

The present study was approved by the institutional review board of Guangdong Provincial People's Hospital and signed informed consent for participation was obtained from the patient and her parents.

Consent for publication

Signed informed consent for publication was obtained from the patient and her parents.

\section{Availability of data and materials}

The datasets used and/or analyzed during the current study are available from the corresponding author on reasonable request.

\section{Competing interests}

The authors declare that they have no competing interests.

\section{Authors' contributions}

Data collection: Rongjie Wu. Drafting manuscript: Rongjie Wu. Revising manuscript content: Guangtao Fu. Approving final version of manuscript: Guangtao Fu. Guangtao Fu takes responsibility for the integrity 
of the data analysis.

\section{Acknowledgments}

Many thanks to Professor Jinguang ye for his consultation.

This work was supported by Science and Technology Program of Guangzhou(202102020050).

\section{REFERENCES}

1. Wong SC, Zacharin M. Long-term health outcomes of adults with McCune-Albright syndrome. Clin Endocrinol (Oxf). 2017;87(5):627-34.

2. Dumitrescu CE, Collins MT. McCune-Albright syndrome. Orphanet J Rare Dis. 2008;3:12.

3. Ippolito E, Bray EW, Corsi A, De Maio F, Exner UG, Robey PG, et al. Natural history and treatment of fibrous dysplasia of bone: a multicenter clinicopathologic study promoted by the European Pediatric Orthopaedic Society. J Pediatr Orthop B. 2003;12(3):155-77.

4. Volkl TM, Dorr HG. McCune-Albright syndrome: clinical picture and natural history in children and adolescents. J Pediatr Endocrinol Metab. 2006;19 Suppl 2:551-9.

5. Narumi S, Matsuo K, Ishii T, Tanahashi Y, Hasegawa T. Quantitative and sensitive detection of GNAS mutations causing mccune-albright syndrome with next generation sequencing. PloS one. 2013;8(3):e60525.

6. Cho EK, Kim J, Yang A, Ki CS, Lee JE, Cho SY, et al. Clinical and endocrine characteristics and genetic analysis of Korean children with McCune-Albright syndrome: a retrospective cohort study. Orphanet J Rare Dis. 2016;11(1):113.

7. Arrigo M, Jessup M, Mullens W, Reza N, Shah AM, Sliwa K, et al. Acute heart failure. Nat Rev Dis Primers. 2020;6(1):16.

8. MacMahon HE. Albright's syndrome-thirty years later. (Polyostotic fibrous dysplasia). Pathology annual. 1971;6:81-146.

9. Shenker A, Weinstein LS, Moran A, Pescovitz OH, Charest NJ, Boney CM, et al. Severe endocrine and nonendocrine manifestations of the McCune-Albright syndrome associated with activating mutations of stimulatory G protein GS. J Pediatr. 1993;123(4):509-18.

10. Landis CA, Masters SB, Spada A, Pace AM, Bourne HR, Vallar L. GTPase inhibiting mutations activate the alpha chain of Gs and stimulate adenylyl cyclase in human pituitary tumours. Nature. 1989;340(6236):692-6.

11. Weinstein LS, Shenker A, Gejman PV, Merino MJ, Friedman E, Spiegel AM. Activating mutations of the stimulatory G protein in the McCune-Albright syndrome. The New England journal of medicine. 1991;325(24):1688-95.

12. Schwindinger WF, Francomano CA, Levine MA. Identification of a mutation in the gene encoding the alpha subunit of the stimulatory G protein of adenylyl cyclase in McCune-Albright syndrome. Proc Natl Acad Sci U S A. 1992;89(11):5152-6.

13. Malchoff CD, Reardon G, MacGillivray DC, Yamase H, Rogol AD, Malchoff DM. An unusual presentation of McCune-Albright syndrome confirmed by an activating mutation of the Gs alpha-subunit from a bone lesion. J Clin Endocrinol Metab. 1994;78(3):803-6.

14. Bianco P, Riminucci M, Majolagbe A, Kuznetsov SA, Collins MT, Mankani MH, et al. Mutations of the GNAS1 gene, stromal cell dysfunction, and osteomalacic changes in non-McCune-Albright fibrous dysplasia of bone. J Bone Miner Res. 2000;15(1):120-8. 
15. Hart ES, Kelly MH, Brillante B, Chen CC, Ziran N, Lee JS, et al. Onset, progression, and plateau of skeletal lesions in fibrous dysplasia and the relationship to functional outcome. J Bone Miner Res. 2007;22(9):1468-74.

16. Collins MT. Spectrum and natural history of fibrous dysplasia of bone. J Bone Miner Res. 2006;21 Suppl 2:P99-P104.

17. Mittal R, Martin RP. Cardiac failure associated with McCune-Albright syndrome. Br J Hosp Med (Lond). 2015;76(10):604-5.

18. Baggish AL, Siebert U, Lainchbury JG, Cameron R, Anwaruddin S, Chen A, et al. A validated clinical and biochemical score for the diagnosis of acute heart failure: the ProBNP Investigation of Dyspnea in the Emergency Department (PRIDE) Acute Heart Failure Score. Am Heart J. 2006;151(1):48-54.

19. Heart Failure Society of A, Lindenfeld J, Albert NM, Boehmer JP, Collins SP, Ezekowitz JA, et al. HFSA 2010 Comprehensive Heart Failure Practice Guideline. J Card Fail. 2010;16(6):e1-194.

20. Packer M, Carver JR, Rodeheffer RJ, Ivanhoe RJ, DiBianco R, Zeldis SM, et al. Effect of oral milrinone on mortality in severe chronic heart failure. The PROMISE Study Research Group. The New England journal of medicine. 1991;325(21):1468-75.

21. Bockus LB, Humphries KM. cAMP-dependent Protein Kinase (PKA) Signaling Is Impaired in the Diabetic Heart. The Journal of biological chemistry. 2015;290(49):29250-8.

22. Ai L, Perez E, Asimes A, Kampaengsri T, Heroux M, Zlobin A, et al. Binge Alcohol Exposure in Adolescence Impairs Normal Heart Growth. Journal of the American Heart Association. 2020;9(9):e015611.

23. Demers C, Rouleau JL, Leung TK, Tardif JC. Hypercalcemic cardiomyopathy associated with primary hyperparathyroidism mimicking primary obstructive hypertrophic cardiomyopathy. Can J Cardiol. 1998;14(11):1397-400.

Figure legends:

Figure 1 Preoperative computed tomography (CT) (A) and Single-photon emission computed tomography (SPECT) imaging (B).

Figure 2 Preoperative design (A) and anteroposterior radiography (B) of the patient after the operation.

Figure 3 Genetic test of the pathological bone tissue from the proximal left femur.

Figure 4 Computed tomography scanning when the patient had dyspnea.
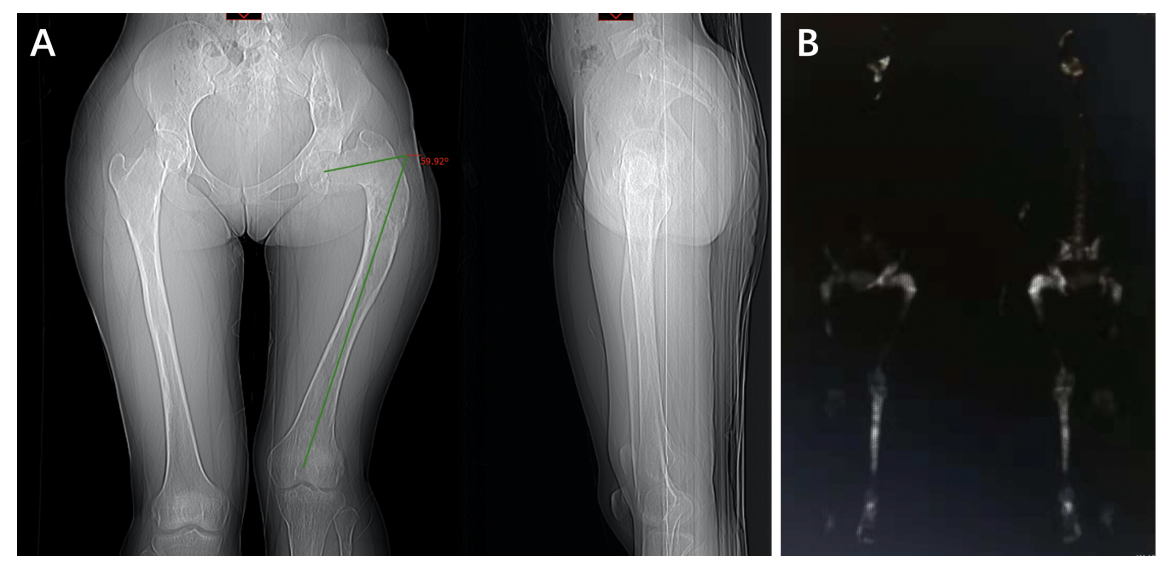

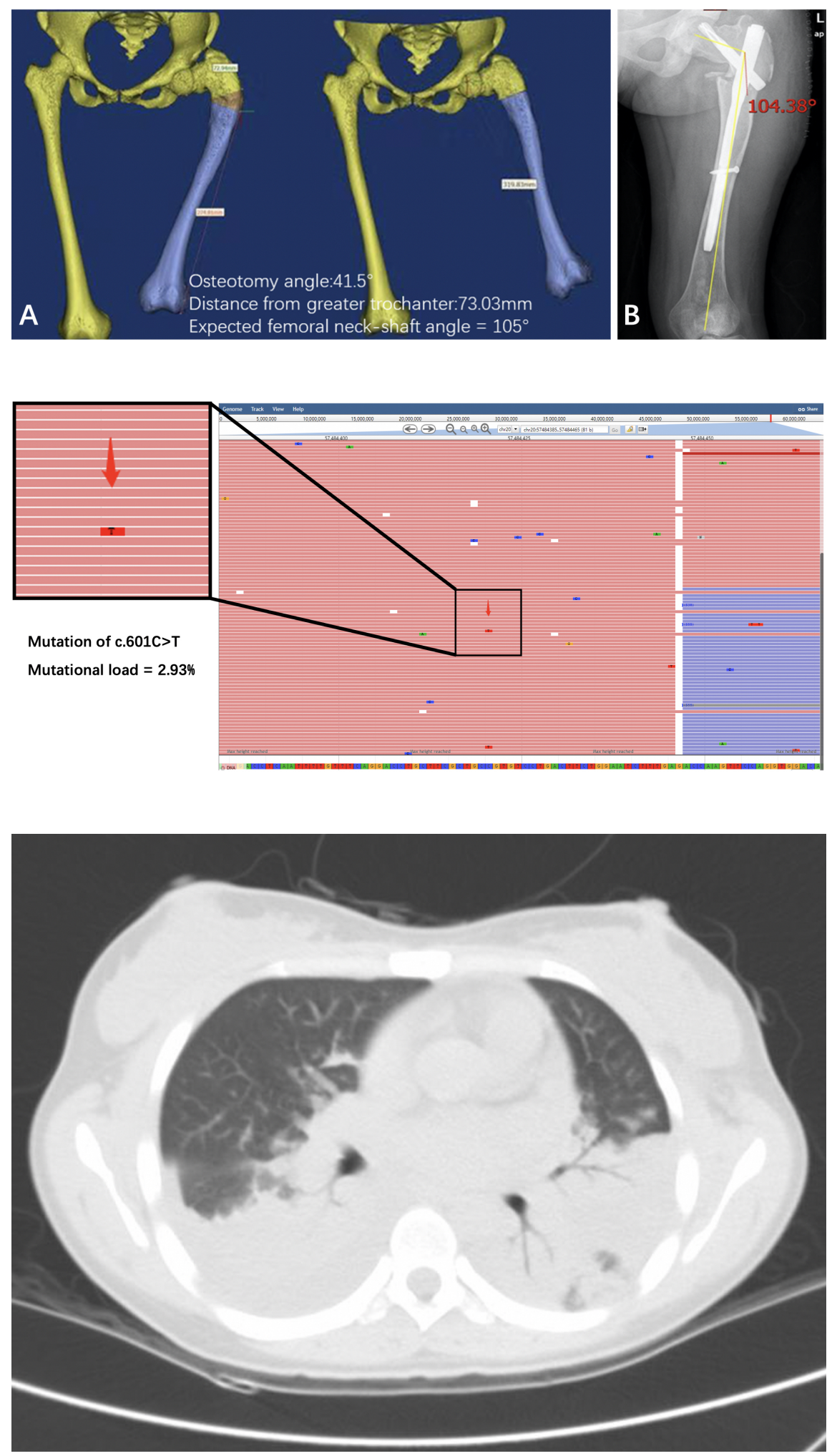